\title{
Cooking related Carbon Footprint Evaluation and Optimisation *
}

\author{
Damien Alvarez de Toledo1[0000-0001-9740-0032] dalvarez@enssat.fr, \\ Laurent d'Orazio3[0000-0001-8614-1848] laurent.dorazio@irisa.fr, \\ Frederic Andres 2[0000-0002-5003-7579] andres@nii.ac.jp, and \\ Maria C.A. Leite ${ }^{40000-0002-8096-7152]}$ mcleite@usf .edu \\ 1 ENSSAT, 22300 Lannion, FRANCE \\ 2 Univ Rennes, CNRS, IRISA, 22300 Lannion, France \\ 3 National Institute of Informatics, Tokyo 101-8430, Japan \\ 4 University of South Florida, St. Petersburg, FL 33701, USA
}

\begin{abstract}
Carbon Footprint of foods has been a major concern for the past few years. Food production is responsible for a quarter of all GHG (Green House Gas) emissions. Many food's Carbon Footprint calculators can be found online but most of them give individual results per ingredient and do not offer a perspective of the whole recipe's Carbon Footprint. Many factors have to be taken into account for this calculation as the origin of the food, the location of the cooker, but also the way to cook and to assemble ingredients. In this paper, we present the CROPPER (CaRbon fOotprint reciPe oPtimizER) that improves an input recipe by updating its ingredients (origin, type) and its cooking procedures to reduce its Carbon Footprint while keeping it savory.
\end{abstract}

Keywords: Carbon Footprint $\cdot$ Recipe $\cdot$ Food $\cdot$ Health $\cdot$ Climate Change

\section{Introduction}

The Earth's atmosphere is warming faster than it probably ever has. In some cases weather patterns, climates and natural environments are changing quicker than wildlife or people can adapt. GHG as carbon dioxide, nitrous oxide and methane form emissions have been recognized as partly responsible for the accelerated rate of the climate change [6].Several reports including standards [5, 10, 11] pointed out the relationship between food consumption and climate change patterns. Also, food production has been recognized to contribute greatly for the anthropogenic environmental disturbances [13]. On the other hand, the choice of our ingredients and ways of cooking has a direct influence on the GHG emissions we produce as citizens (e.g., $[16,18])$. Thus, it is important to increase our awareness on both how our food can contribute towards the problem of climate change, and how climate change threatens the supply of ingredients we take for

^ D. Alvarez de Toledo has been a research student at NII during the research period that led to the production of this paper. 
granted. In particular, a conscious approach to the choice of ingredients is vital to reduce GHC and, consequently, our personal environmental impact.The concept of food Carbon Footprint is being increasingly studied by researchers around the world. It has not yet reached, however, a high awareness amongst consumers. The general public as well as specialised chefs need a concise and simple method to evaluate their cooking GHG emissions and fulfilling this awareness. Most information around food's carbon footprint is detailed for separate ingredients and does not take into account the impact that their combination can have as a recipe $[3,6,15]$. Our research, part of the CRWB project [1] aims to overcome this boundary and to assist reducing the individual's Carbon Footprint when cooking a specific recipe, by submitting the recipe to a Carbon footprint optimizer algorithm, the CROPPER.

This paper is structured as follows. Section 2 reviews the state of art. Section 3 describes the Low Carbon Footprint recipe optimiser and our implementation choices. Section 4 introduces the preliminary results. Finally, Section 5 concludes and introduces the future works.

\section{State of the art}

The concept of Carbon Footprint (CF) appeared around 2006, but the climate impact of products has been calculated for decades as part of full Life cycle assessment(LCA) [15]. Numerous papers about the emitted CF of individual and specific ingredients (e.g., $[15,17]$ and references therein), but also Danish diets [2] and foods life-cycle in Finnish households [12] are accessible nowaday. However, the computation of $\mathrm{CF}$ is still a challenging endeavor. Availability of online CF calculators is quite recent and they focus on single specific ingredients only $[3,6]$. Some other calculators try to offer an opportunity for the user to optimize a recipe's environmental impact by swapping ingredients, but they lack an interesting variety of ingredients in their database and are designed for outof-home catering sector [17]. They are not yet available for private households and their design is in a single language only, not extended to English, which limit their usage by general public worldwide. Furthermore, the available online calculator do not incorporate the $\mathrm{CF}$ and budget optimizer feature that is been built into CROPPER. Previous works can be considered as stepping stones for an upcoming system that can evaluate the $\mathrm{CF}$ of a recipe in a complete manner, the CROPPER, that "crops" the Carbon Footprint (CF) of an input recipe to make it more environmentally friendly. This system will contain a multilingual service and database, including most European and Asian languages.

\section{CROPPER Model}

\subsection{CROPPER theoretical Approach}

The following theoretical approach aims to reduce the Carbon Footprint (named $C F$ ) of an input recipe by considering a Desired Carbon Footprint (named DCF) 
and a Money Threshold, both entered by the user. We assume the ingredient similarity between the input and output recipe for this research. When the ingredient is swapped, it can only be replaced by an ingredient from the exact same kind i.e. a banana by another banana (different origin, organic instead of conventional, etc.).

We define the output_recipe_CF, the CF estimated for the output recipe, as follows:

output_recipe_CF $=\sum_{i=1}^{\text {nb_Ingredients }} C F($ ingredient_i $), \quad$ output_recipe_CF $\leq D C F$

where nb_Ingredients and $\mathbf{C F}$ (ingredient_i) denotes, respectively, the number of ingredients and the price of the ith ingredient in the recipe.

If DCF cannot be attained through ingredient swapping, then the closest output_recipe_CF to DCF will be returned jointly to the updated recipe. The price of the processed recipe (recipePrice) must respect the condition of being inferior or equal to the user's budget (Money_Threshold) as follows :

recipePrice $=\sum_{i=1}^{\text {nb_Ingredients }}$ Price $\left(\right.$ ingredient $\left._{i}\right), \quad$ recipePrice $\leq$ Money_Threshold,

where Money_Threshold is the budget of the User and Price(ingredient_i) is the price of the ith ingredient of the recipe. The CaRbon fOotprint reciPe oPtimizER (CROPPER) ecosystem of this study is represented in (Fig. 1a). The user selects a recipe and a desired $\mathrm{CF}$ value as an input in addition to

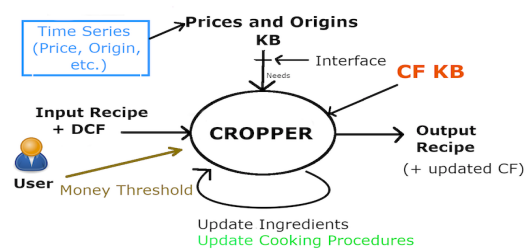

(a)

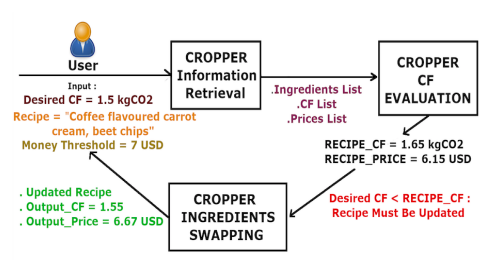

(b)

Fig. 1: (a) CROPPER Ecosystem schematic representation. (b) Use-case result tracing.

a money threshold, which corresponds to the user's budget. Two conditions must be met for the recipe to be updated (ingredients swapping): 1) The user must provide the required amount of money for swapping. 2) The recipe will be updated when its current $\mathrm{CF}$ is higher than the desired one, for a given budget. The final goal of this research is to develop a service that interacts with two knowledge bases FoodPriceBazaar ${ }^{5}$ and CFKB. The former (Prices and Origins KB in Fig. 1a) contains information related to a product's shop, price

\footnotetext{
5 https://bit.ly/3cF7hP7
} 
and origin. The latter (CF KB in Fig. 1a) provides data on each ingredient's $\mathrm{CF}$ according to their origin. Both of the knowledge bases will be design so that, as opposed to the existing one, each ingredient will have its own semantic signification. Thus, making them more suitable for use compared to raw data included in a conventional database.

\subsection{CROPPER pseudo-code implementation}

The key parts of the CROPPER algorithms are (1) the evaluation of the actual Carbon Footprint (CF) and the cost of the recipe CF-Evaluation() in Algorithm 1, and (2) the CF reduction accomplished through the use of IngredientsSwapping () according to the user's requirements in Algorithm 2.

By default the first ingredients retrieved by Information-Retrieval() algorithm, which is not the focus of this article and, therefore, its description it is not included, are the ones that are geographically closest to the cooker's location (the distance is given in $\mathrm{km}$ ). The implicit assumption is that these ingredients have a smaller CF. This may not always be true as it has been shown in some recent studies [18]. However, the United Nations recommend to 'Eat local' aiming to reduce food related $\mathrm{CF}$. As this is a first developed system that can evaluate and optimise the $\mathrm{CF}$ of a recipe, we made this assumption as a starting point. Its relaxation is seen as a future study.
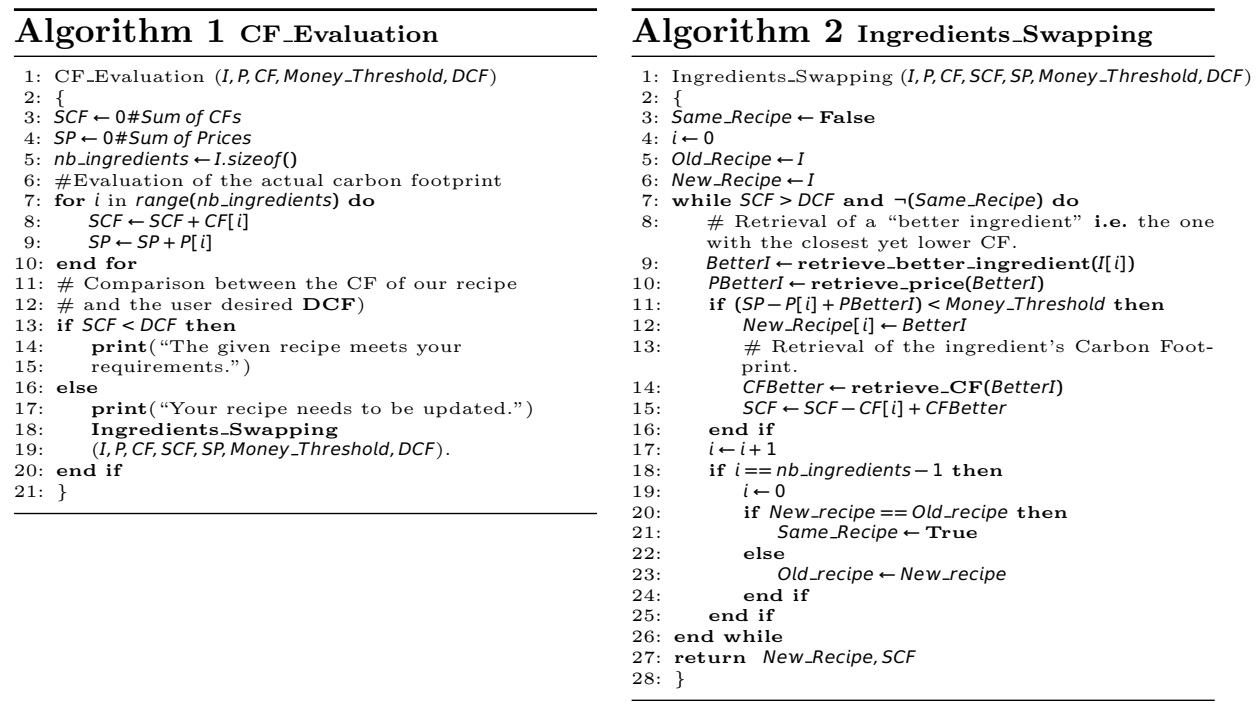

Observe that if DCF cannot be attained by the algorithm Ingredients_Swapping, the condition Old_Recipe $==$ New_Recipe must be met for the algorithm to stop. The number of ingredients in the database (either the Dummy Database or the future $\mathrm{KB}$ ) is finite and the choice of the ingredients in the algorithm is always oriented towards a "better" one (lower yet closest CF compared to the previous ingredient). We can thus infer that it is not possible to go back to choosing an ingredient with a higher CF and New_Recipe is a loop variant. When New_Recipe 
cannot change anymore (no "better" ingredients left in the database) redundancy is met with Old_Recipe. The algorithm then stops.

The Knowledge Bases FoodPriceBazaar and CFKB are yet not available. Hence, the study was conducted on a dummy database created by retrieving information from distinct sources for ingredients $\mathrm{CF}[4,8,9,14]$ and prices ${ }^{6,7}$.

\section{Implementation and Results}

Our use-case was coded in Python, whilst the dummy database presents three different origins for each ingredient e.g. Sour Cream from France, USA or Argentina. The code implementation and the dummy database are available on bitbucket ${ }^{8}$. A study was performed to determine whether it is possible to develop an algorithm that can swipe ingredients in a given recipe aiming to reduce its CF with a pre-specified budget (in USD). The Use-Case used in this study was the dish: "Coffee flavoured carrot cream, beet chips" (see Fig. 1b). After launching our three sub-algorithms altogether, the default recipe is firstly determined with the cooker's location accordingly. We then obtained an update of our recipe with a lower $\mathrm{CF}\left(1.65 \mathrm{kgCO}_{2}\right.$ to $\left.1.55 \mathrm{kgCO}_{2}\right)$ yet a higher price (6.15 USD to 6.67 USD). The results of our first implementation remain substantially simplistic in the sense that the choice of variety for each ingredient is not very broad in our dummy database (three for each). More significant results will be obtained when the connection with our two Knowledge Bases (Prices and $\mathrm{CF}$ ) is materialized.

\section{Conclusion and Future Work}

Climate change is happening at high speed and has many origins, and our diet is one of them. Developing models to reduce our environmental impact when cooking/eating is thus a valuable goal. In this study, we present a Carbon Footprint $(\mathrm{CF})$ recipe optimiser which has a number of benefits over existing calculators. Current $\mathrm{CF}$ calculators for foods focus on one ingredient at a time and thus lack the assembling feature for getting the environmental impact of a whole recipe.

The findings in this paper can be used as a base for a better recipe updater that takes into account ingredients pairing and also the proper connectivity to the relevant knowledge bases. The latter will provide a large scope of ingredients variety. Future features are its scalability and bulking, allowing the simultaneous feeding of many recipes as an input, which will yield more diversified results.

Our current model and preliminary tests show that it is possible to compute a new recipe given a certain money budget and a desired CF. Thus, it satisfy our query for a better $\mathrm{CF}$ for an input recipe. This novel study has the advantage

\footnotetext{
${ }^{6}$ Walmart, www.walmart.com. Last accessed 25/4/2020

7 Climate change food calculator: What's your diet's carbon footprint?, www.bbc.com/news/science-environment-46459714. Last accessed 23/4/2020

8 https://bit.ly/2zbpQNe
} 
of proposing a clear design of a recipe CF optimiser, easily understood and visualized through the pseudo-code and model exposed. Although the process is limited to summations for now, it offers the capability of processing a whole recipe at once, and not forcing the user to enter each ingredient with its respective volume. The three main limitations of our approach is the transport $\mathrm{CF}$ of ingredients, find eco-friendly cooking procedures, and the access to the target knowledgebases have not yet fully implemented. Additionaly, to improve the quality of the optimised recipes, the Ingredient pairing feature will be included.

\section{Acknowledgements}

We would like to express our deepest appreciation to the National Institute of Informatics for the ongoing research support.

\section{References}

1. F. Andres, The CRWB RSbench: Towards a Cooking Recipe Benchmark Initiative, 2018 IEEE ICDE Workshops, 2018, pp. 154-156, 10.1109/ICDEW.2018.00032.

2. Bruno, M. et al.: The carbon footprint of Danish diets. Climatic Change. 156, (2019)

3. Carbon Food Calculator-The Vegan Society, https://bit.ly/2Xwo5U2. Last accessed $29 / 4 / 20$

4. Chart: The Carbon Footprint of the Food Supply Chain, https://bit.ly/2MtlDHx. Last accessed 25/04/20

5. Clark, M. et al.: Multiple health and environmental impacts of foods, Proceedings of the National Academy of Sciences 116 (2019)

6. Climate Change Indicators: Greenhouse Gases, https://www.epa.gov/climateindicators/greenhouse-gases. Last accessed 29/04/20

7. Coffee's Invisible Carbon Footprint, https://bit.ly/2U9Yoqi. Last accessed 25/04/20

8. Do you know the carbon footprint of these common foods, https://bit.ly/2Xwp06W. Last accessed 25/04/20

9. Émissions indirectes - autres, Restauration, Repas, https://bit.ly/2AJZcew. Last accessed 25/04/20

10. Folk and Knife: Food \& CO2, What are the carbon emissions of different foods? https://folkandknife.com/wp-content/uploads/2019/05/Food-CO2-2.pdf (2019)

11. Guide to PAS 2050, How to assess the carbon footprint of goods and services, British Standard PAS 2050, pp58, ISBN 978-0-580-64636-2, 2008

12. Kauppinen, T. et al.: Carbon footprint of food-related activities in Finnish households. Progress in Industrial Ecology An International Journal. 7, 257 - 267 (2010)

13. Poore, J., Nemecek, T.: Reducing food's environmental impacts through producers and consumers. Science 360(6392), 987-992 (2018)

14. Réduire sa consommation de sel: pourquoi?, https://bit.ly/3aERwXk. 25/04/20

15. Röös, E.: Analysing the Carbon Footprint of Food. Acta Universitatis Agriculturae Sueciae (1652-6880) 2013(56), 0-96 (2013)

16. Sandström, V. et al.: The role of trade in the greenhouse gas footprints of EU diets. Global Food Security 19, 48-55 (2018)

17. Speck, M. et al.: Creating Sustainable Meals Supported by the NAHGAST Online Tool - Approach and Effects on GHG Emissions and Use of Natural Resources. Sustainability, (12)(1136) (2020)

18. Weber, C.L., Matthews, S.: Food-Miles and the Relative Climate Impacts of Food Choices in the United States. Environ. Sci. Technol. 42(10), 3508-3513 (2008) 